\title{
Applications of artificial intelligence to prognostic stratification of COVID-19: a narrative review
}

\author{
Elena Prisciandaro ${ }^{1}$, Luca Bertolaccini $^{1 \wedge}{ }^{\text {, Lorenzo Spaggiari }}{ }^{1,2}$ \\ ${ }^{1}$ Department of Thoracic Surgery, IEO, European Institute of Oncology IRCCS, Milan, Italy; ${ }^{2}$ Department of Oncology and Hemato-Oncology, \\ University of Milan, Milan, Italy \\ Contributions: (I) Conception and design: L Bertolaccini; (II) Administrative support: L Spaggiari; (III) Provision of study materials or patients: \\ E Prisciandaro; (IV) Collection and assembly of data: L Bertolaccini, E Prisciandaro; (V) Data analysis and interpretation: L Bertolaccini, E \\ Prisciandaro; (VI) Manuscript writing: All authors; (VII) Final approval of manuscript: All authors. \\ Correspondence to: Luca Bertolaccini, MD, PhD, FCCP. Division of Thoracic Surgery, IEO, European Institute of Oncology IRCCS, Via Ripamonti \\ 435 - 20141 Milan, Italy. Email: luca.bertolaccini@gmail.com.
}

\begin{abstract}
Objective: New approaches incorporating artificial intelligence solutions have proven successful and valuable support for decision-making. The purpose of this review is to describe the emerging artificial intelligence applications to the prognostic stratification and profiling of patients suffering from COVID-19.

Background: COVID-19 has become a public health emergency, alarming social and economic impact on healthcare systems worldwide. It is paramount to identify patients at the highest risk of developing severe COVID-19, thus improving resource allocation.

Methods: A systematic literature search for articles published in English between the date of database inception and January 31, 2021, was performed in EMBASE (via Ovid), MEDLINE (via PubMed) and Cochrane CENTRAL.

Conclusions: Several artificial intelligence-based approaches have been conceived to ease the pressure on the overloaded health system and assist clinicians in the prognostic profiling of COVID-19 patients. Risk assessment and categorisation are essential: by identifying the more likely subjects to suffer from an acute disease, it might be possible to plan a closer monitoring and/or earlier therapeutic intervention. Hence, artificial intelligence (AI) may support physicians in adjusting their management strategy according to the prognostic estimation, resulting in improved quality of care. This would also facilitate resource allocation in a time when careless supply distribution is not allowed. Artificial intelligence may support physicians in adjusting their management strategy according to the prognostic estimation, resulting in improved quality of care.
\end{abstract}

Keywords: Coronavirus-induced disease (COVID-19); artificial intelligence (AI); narrative review

Received: 10 July 2021; Accepted: 23 November 2021; Published: 30 January 2022.

doi: $10.21037 /$ shc-21-17

View this article at: https://dx.doi.org/10.21037/shc-21-17

\section{Introduction}

In late 2019, a series of pneumonia cases of unknown cause was reported in Wuhan, China, leading to the identification of a new pathogen, named 2019 novel Coronavirus (2019$\mathrm{nCoV}$ ) or severe acute respiratory syndrome Coronavirus
2 (SARS-CoV-2) $(1,2)$. Following the global spread of SARS-CoV-2 infection, the Coronavirus-induced disease (COVID-19) was declared a pandemic by the World Health Organization (3). Over 76 million confirmed cases and more than 1.6 million deaths have been registered in 190 countries, and numbers continue to increase (4).

^ ORCID: 0000-0002-1153-3334. 
COVID-19 has become a public health emergency, with an alarming social and economic impact on healthcare systems worldwide. Early detection of SARS-CoV-2 infection is crucial to interrupt virus transmission to close contacts and adequate patient monitoring and worsening clinical prevention. Besides, due to understaffing and patient overload in healthcare facilities, it is paramount to identify patients at the highest risk of developing severe COVID-19, thus improving resource allocation. New approaches incorporating artificial intelligence (AI) solutions have proven successful and valuable support for decision-making (5-7). The purpose of this review is to describe the emerging $\mathrm{AI}$ applications to the prognostic stratification and profiling of patients suffering from COVID-19. We present the following article in accordance with the Narrative Review reporting checklist (available at https://shc.amegroups.com/ article/view/10.21037/shc-21-17/rc).

\section{Materials and methods}

A systematic literature search for articles published in English between the date of database inception and January 31, 2021, was performed in EMBASE (via Ovid), MEDLINE (via PubMed) and Cochrane CENTRAL. Single-arm case series, case reports, letters, editorials, and expert opinions were not considered. Records identified through the designed search strategy were imported into reference management software. In case of duplication, the most recent paper was selected. Two reviewers (EP and LB) independently screened the titles and abstracts of all the imported articles. Full-text copies of potentially eligible reports were separately evaluated by the same reviewers (EP and LB). When multiple studies contained overlapping data, the most informative one was included. Any disagreements were resolved by consensus or arbitration by a third investigator (LS).

\section{Analysis of the literature}

Starting from a large, computed tomography (CT) dataset of SARS-CoV-2-induced pneumonia, Zhang and colleagues (8) developed an AI system to support radiologists and clinicians in the early identification of patients at a higher risk of developing the critical illness, which was defined as a clinical condition requiring admission to the Intensive Care Unit (ICU) and/or mechanical ventilation, or death. Clinical prognosis estimation was mainly influenced by the features of the lung lesions on CT scans. There was a positive correlation between the lesion characteristics and the degree of organ damage. The proposed AI model, which combined CT findings and clinical data, showed a specificity of $80.00 \%$ and sensitivity of $86.71 \%$.

Similarly, Chassagnon et al. (9) presented a disease severity assessment method using an ensemble learning approach. In particular, the authors aimed at anticipating patient outcomes before the mechanical ventilation requirement. They elaborated a holistic signature incorporating radiological descriptors, biological and clinical data from a multicentre dataset.

Izquierdo and colleagues (10) conducted a multicentre retrospective study of the electronic records of 10,504 patients with COVID-19. They developed a machinelearning algorithm to classify the patients according to their risk of being admitted to the ICU. Based on their analysis, a combination of three easily available clinical variables (age, temperature, and respiratory frequency) was the most parsimonious predictor of ICU admission. The reported model showed accuracy, recall, and area under the curve values of $0.68,0.71$, and 0.76 , respectively.

Mushtaq et al. (11) compared AI- and radiologist-assessed severity scores calculated on chest X-rays of 697 patients presenting at the emergency department with SARS-CoV-2 infection. The AI system was based on convolutional neural networks and Natural Language Processing trained to detect specific abnormalities on frontal Roentgenograms. The authors disclosed similar performances of the two models, which were also found to be independent outcome predictors in multivariate regression models, including age, sex, and comorbidities. Interestingly, both scores were significantly higher in patients with symptoms for $\geq 7$ days $(\mathrm{P}=0.031$ and 0.014$)$ and peripheral predominance of lung opacities was the only significantly different radiographic finding between the two groups $(\mathrm{P}=0.001)$.

Abdulaal and colleagues (12) built and trained an artificial neural network to examine several COVID-19 patient characteristics (demographics, comorbidities, lifestyle factors, and presenting symptoms) to predict patientspecific mortality risk. The sensitivity and specificity of this model were $87.50 \%$ and $85.94 \%$, respectively.

\section{Comment}

Hospitals worldwide are facing extreme difficulties during 
the COVID-19 pandemic. Healthcare providers are confronted with increasing numbers of infected people, a shortage of ICU beds and medical stocks, and nonnegligible risks of self-contamination. Several AI-based approaches have been conceived to ease the pressure on the overloaded health system and assist clinicians in the prognostic profiling of COVID-19 patients (13). Risk assessment and categorisation are essential: by identifying the more likely subjects to suffer from an acute disease, it might be possible to plan a closer monitoring and/or earlier therapeutic intervention. Hence, AI may support physicians in adjusting their management strategy according to the prognostic estimation, resulting in improved quality of care. This would also facilitate resource allocation in a time when careless supply distribution is not allowed.

In conclusion, AI solutions might represent a quick, costeffective, and reliable tool for predicting disease severity in COVID-19 patients, time-saving but also lifesaving.

\section{Acknowledgments}

Funding: None.

\section{Footnote}

Reporting Checklist: The authors have completed the Narrative Review reporting checklist. Available at https:// shc.amegroups.com/article/view/10.21037/shc-21-17/rc

Conflicts of Interest: All authors have completed the ICMJE uniform disclosure form (available at https://shc.amegroups. com/article/view/10.21037/shc-21-17/coif). LB and LS serve as unpaid editorial board members of Shanghai Chest. The other author has no conflicts of interest to declare.

Ethical Statement: The authors are accountable for all aspects of the work in ensuring that questions related to the accuracy or integrity of any part of the work are appropriately investigated and resolved.

Open Access Statement: This is an Open Access article distributed in accordance with the Creative Commons Attribution-NonCommercial-NoDerivs 4.0 International License (CC BY-NC-ND 4.0), which permits the noncommercial replication and distribution of the article with the strict proviso that no changes or edits are made and the original work is properly cited (including links to both the formal publication through the relevant DOI and the license). See: https://creativecommons.org/licenses/by-nc-nd/4.0/.

\section{References}

1. Guan WJ, Ni ZY, Hu Y, et al. Clinical Characteristics of Coronavirus Disease 2019 in China. N Engl J Med 2020;382:1708-20.

2. Huang C, Wang Y, Li X, et al. Clinical features of patients infected with 2019 novel coronavirus in Wuhan, China. Lancet 2020;395:497-506.

3. World Health Organization. Coronavirus disease (COVID-19) outbreak. Available online: https://www. who.int

4. Available online: https://www.worldometers.info/ coronavirus/.

5. Chang AC. Artificial Intelligence and COVID-19: Present State and Future Vision. Intell Based Med 2020;3:100012.

6. Lalmuanawma S, Hussain J, Chhakchhuak L. Applications of machine learning and artificial intelligence for Covid-19 (SARS-CoV-2) pandemic: A review. Chaos Solitons Fractals 2020;139:110059.

7. van der Schaar M, Alaa AM, Floto A, et al. How artificial intelligence and machine learning can help healthcare systems respond to COVID-19. Mach Learn 2020. doi: 10.1007/s10994-020-05928-x.

8. Zhang K, Liu X, Shen J, et al. Clinically Applicable AI System for Accurate Diagnosis, Quantitative Measurements, and Prognosis of COVID-19 Pneumonia Using Computed Tomography. Cell 2020;181:14231433.e11.

9. Chassagnon G, Vakalopoulou M, Battistella E, et al. AI-driven quantification, staging and outcome prediction of COVID-19 pneumonia. Med Image Anal 2021;67:101860.

10. Izquierdo JL, Ancochea J; Savana COVID-19 Research Group, et al. Clinical Characteristics and Prognostic Factors for Intensive Care Unit Admission of Patients With COVID-19: Retrospective Study Using Machine Learning and Natural Language Processing. J Med Internet Res 2020;22:e21801.

11. Mushtaq J, Pennella R, Lavalle S, et al. Initial chest radiographs and artificial intelligence (AI) predict clinical outcomes in COVID-19 patients: analysis of 697 Italian patients. Eur Radiol 2021;31:1770-9.

12. Abdulaal A, Patel A, Charani E, et al. Prognostic Modeling 
of COVID-19 Using Artificial Intelligence in the United Kingdom: Model Development and Validation. J Med Internet Res 2020;22:e20259.

13. Soltan AAS, Kouchaki S, Zhu T, et al. Rapid triage

doi: $10.21037 /$ shc-21-17

Cite this article as: Prisciandaro E, Bertolaccini L, Spaggiari L. Applications of artificial intelligence to prognostic stratification of COVID-19: a narrative review. Shanghai Chest 2022;6:4. for COVID-19 using routine clinical data for patients attending hospital: development and prospective validation of an artificial intelligence screening test. Lancet Digit Health 2021;3:e78-87. 